\title{
9 Konferenzdolmetschen@ZHAW: Über Kursinhalte, Qualitätssicherung, Praxisnähe und Wissenschaftsbasiertheit
}

\begin{abstract}
Mit der Einführung der Masterstudiengänge hat die Ausbildung von Studierenden zu KonferenzdolmetscherInnen und damit professionellen mehrsprachigen KommunikationsdienstleisterInnen einen wichtigen Entwicklungsschritt vollzogen. Zum einen erfuhr die Ausbildung eine theoretisch-empirische Fundierung durch die Einbettung in den Masterstudiengang Angewandte Linguistik, zum anderen wurde das Curriculum der Vertiefung Konferenzdolmetschen weiter diversifiziert und professionalisiert. Die Erkenntnisse aus der interdisziplinären Forschung werden kombiniert mit praxisrelevanter Problemlösekompetenz in der mehrsprachigen Kommunikation und einer Befähigung zum professionellen Marktauftritt.
\end{abstract}

Die Hochschulreformen im Rahmen des Bologna-Prozesses haben eine Reihe von Veränderungen angestossen, die auch die translatorischen Studiengänge nachhaltig betreffen (Kalina, 2017). Ein Beispiel für eine besonders konsequente Weiterentwicklung eines solchen Studiengangs ist die Ausbildung in Konferenzdolmetschen an der ZHAW Zürcher Hochschule für Angewandte Wissenschaften. Die vorgängig rein auf das Erlernen praktischer Fähigkeiten ausgerichtete Ausbildung der ehemaligen Dolmetscherschule Zürich (DOZ) wurde im Zuge der Einführung eines Masters in Angewandter Linguistik 2009 durch theoretische Fächer ergänzt, die den studierenden DolmetscherInnen und angehenden KommunikationsexpertInnen den wissenschaftlichen Hintergrund ihrer Tätigkeit vermitteln. Dabei wurde der Anspruch einer forschungsgeleiteten Lehre in einer stark praxisorientierten Ausbildung durch die Schaffung einer Professorenstelle für Dolmetschwissenschaft (2011) unterstrichen, die eng mit der Vertiefungsleitung Konferenzdolmetschen zusammenarbeitet. 
Zusätzlich zu strukturellen Veränderungen wurde die Vertiefung Konferenzdolmetschen aber auch inhaltlich laufend den heutigen Anforderungen an KonferenzdolmetscherInnen angepasst. Nebst der Erschliessung neuer Kursinhalte wurden die Unterrichtsformen - wo sinnvoll - mit E-Learning-Angeboten ergänzt, speziell für Dolmetschdozierende Weiterbildungskurse sowie Team-Teaching zur stärkeren Vernetzung und Qualitätssicherung eingeführt, ein praxisnahes Format für Abschlussprüfungen entwickelt und der Austausch mit zukünftigen ArbeitgeberInnen für KonferenzdolmetscherInnen sowie etablierten MarktakteurInnen national und international verstärkt. Freiwillige Dolmetscheinsätze der Studierenden unter Anleitung der Vertiefungsleitung runden ein mittlerweile breit abgestütztes Ausbildungsangebot ab.

\section{Neue Kurse und Kursinhalte}

Während die Studierenden im ordentlichen Dolmetschunterricht die Dolmetschtechniken anhand spezifischer Sprachrichtungen trainieren (z.B. FRA-DEU), bietet sich ihnen im Fach Konferenzsimulation die Möglichkeit, die erlernten Strategien sprachübergreifend anzuwenden. Mit Hilfe von authentischem Bildund Tonmaterial üben die Studierenden reale Dolmetschsituationen in längeren Unterrichtsblöcken (3 Std.). Um den Erfahrungshorizont für die Studierenden zusätzlich zu erweitern, wird dieser Kurs seit einigen Jahren im Team-Teaching geführt. Aufgrund der verschiedenen in den Dolmetschklassen vertretenen Muttersprachen ist somit auch die Anwesenheit von Dozierenden mit anderen Muttersprachen als Deutsch unerlässlich. Der Team-Teaching-Ansatz erweist sich in mehrfacher Hinsicht als wertvoll: Zum einen ergänzen sich die Dozierenden in ihren vielfältigen Erfahrungen auf dem Dolmetschermarkt. Andererseits liefern sie ein vielfältigeres Feedback, als dies im Unterricht mit einer Lehrperson möglich wäre. Schliesslich fördert der gemeinsame Unterricht den Austausch sowie die Abstimmung unter den Dozierenden und wirkt somit qualitätssteigernd.

Während früher jeder/jede Dozierende eine eigene Einführung in die Simultantechnik entwickeln musste, finden heute gemeinsame, sprachenpaarübergreifende Einführungsveranstaltungen ins Simultandolmetschen (seit 2012) sowie für Dolmetschen mit Text (seit 2013) statt. Die Studierenden erhalten so eine strukturierte Anleitung zu den verschiedenen Dolmetschtechniken, die während der folgenden drei Semester im Unterricht eingeübt und vertieft werden. Die einzelnen Dozierenden können auf diesen Grundlagen aufbauen und dadurch schneller zu anspruchsvolleren Übungen übergehen. Diese gemeinsamen Workshops jeweils 
zu Semesterbeginn (im Dolmetsch-Propädeutikum sowie im 1. und 2. Semester) wirken bei den Studierenden zudem identitätsstiftend - ein für den Lernfortschritt und die Persönlichkeitsentwicklung nicht zu unterschätzender Faktor.

DolmetscherInnen werden in erster Linie über die Stimme wahrgenommen. Sie muss über mehrere Jahrzehnte als Trägermedium für die Dolmetschleistung funktionieren. Aufgrund des engen Zeitbudgets ist im sprachenpaarspezifischen Unterricht eine gründliche Auseinandersetzung mit dem Sprechapparat jedoch nicht möglich. Abhilfe schafft hier der 2013 eingeführte Kurs in Stimmbildung, in dem die Studierenden lernen, ihre Stimme als formbares Werkzeug zu begreifen und in den grösseren Zusammenhang anderer Körperfunktionen (insbesondere der Atmung) zu stellen. Durch den Beizug eines externen Fachmanns, der sonst für die Schulung von SprecherInnen vor und hinter der Fernsehkamera zuständig ist, bringen wir ganz bewusst eine Sicht von aussen in unser Studium. Für die Studierenden ist der Ansatz eines Unterrichts ohne Textmaterial, dafür mit Übungen zur körperbezogenen Achtsamkeit zuerst eine Überraschung, im Kursverlauf dann aber geradezu eine Offenbarung.

In eine ähnliche Richtung gehen 2015 neu eingeführte, eintägige Workshops in Stressmanagement, die als Vorbereitung auf die Eignungs- und Abschlussprüfungen angeboten werden. Die Studierenden lernen, mit Drucksituationen vor Dolmetschprüfungen und allenfalls später im Berufsleben mit anspruchsvollen Situationen besser umzugehen, indem sie (Prüfungs-)Angst erkennen, annehmen, systematisch reflektieren, kontrollieren und darüber hinwegkommen können. Die Rückmeldungen der Studierenden aus den ersten Durchführungen der Kurse waren überwältigend positiv.

Damit die Studierenden nicht nur in technischen Fertigkeiten ausgebildet werden, sondern auch über einen professionellen Marktauftritt Bescheid wissen, gab es in der Ausbildung für Konferenzdolmetscher schon länger einen Kurs in Berufskunde. Dieser Kurs wurde vor einigen Jahren ebenfalls für die Vertiefung Fachübersetzen eingeführt und um das Thema Selbständigkeit erweitert. Damit sind die AbsolventInnen beider Vertiefungen in der Lage, sich von Anfang an in einem immer komplexeren Markt zurechtzufinden. Sie kennen die für eine Selbständigkeit erforderlichen gesetzlichen Grundlagen und administrativen Auflagen, können ihre Dienstleistung kompetent vermarkten und sich mit BerufskollegInnen effizient vernetzen. Dazu pflegt die ZHAW den direkten Kontakt zu den Standesorganisationen DÜV (Dolmetscher- und Übersetzervereinigung) und ASTTI (Schweizerischer Übersetzer-, Terminologen- und Dolmetscher-Verband).

Neu eingeführt wurde eine simulierte ELF-Konferenz, welche die im Zuge der Globalisierung rasant zunehmende Entwicklung einer wachsenden Zahl von nichtmuttersprachlichen EnglischrednerInnen auf Konferenzen aufgreift. Die im 
Rahmen der Professorenstelle geleistete Pionierarbeit im Bereich ITELF (Interpreting, Translation and English as a Lingua Franca) (Albl-Mikasa, 2017) fand hier ihre unmittelbare Umsetzung. Ein über drei Semester erfolgreich durchgeführtes, wissenschaftlich begleitetes Pilotprojekt verweist auf die Bedeutung von systematischen Übungs- und Sensibilisierungseinheiten, in denen die Dolmetschstudierenden Strategien und Kompetenzen im Umgang mit dieser in der beruflichen Praxis auf sie zukommenden Realität entwickeln können (AlblMikasa et al., 2017). Nachdem die ELF-bezogenen Einheiten auch bei den Studierenden auf grossen Zuspruch gestossen sind, werden sie weiter ausgearbeitet und fortgesetzt.

\section{Videokonferenzen und Rededatenbank}

Die technologische Entwicklung eröffnet auch im Konferenzdolmetschen neue Möglichkeiten. An der ZHAW wird deshalb seit einigen Jahren ein besonderes Unterrichtsformat praktiziert, sogenannte Virtual Master Classes, die zweimal pro Semester stattfinden. Dabei handelt es sich um Lektionen, die mittels VideoKonferenzsystem gemeinsam mit professionellen DolmetscherInnen beim EUParlament und der EU-Kommission in Brüssel durchgeführt werden. Auf Seiten der ZHAW sitzen jeweils alle Studierenden eines Jahrgangs, in Brüssel sind es fünf bis sechs professionelle EU-DolmetscherInnen. Im Verlauf der Fernlektion halten die Kollegen in Brüssel jeweils kurze Reden (5-8 Minuten) in den vereinbarten Sprachen, die von den Studierenden aus Winterthur konsekutiv gedolmetscht werden. Die Rückmeldung kommt dann wiederum direkt aus Brüssel. Die Studierenden erfahren so, wie ihre Leistung ausserhalb der ZHAW von qualifizierten KollegInnen bewertet würde. Im Anschluss an die Virtual Master Class besprechen die Dozierenden der ZHAW mit den Studierenden die Erkenntnisse aus der Fernlektion. Dieser direkte Kontakt zu einem zukünftigen Arbeitgeber ist für die Ausbildung äusserst gewinnbringend: Bereits der kurze Blick von aussen während der Ausbildung bewirkt bisweilen noch mehr als die regelmässigen Feedbacks der vertrauten Dozierenden.

Die Arbeit mit hochwertigem Übungsmaterial spielt auch in der Dolmetscherausbildung eine zentrale Rolle. Mit den heutigen elektronischen Lehrplattformen eröffnete sich hier eine neue Möglichkeit: Für Prüfungen werden stets Dutzende von Reden aufbereitet und seit 2013 als Videos vorgängig aufgezeichnet. Damit dieses wertvolle Material nicht nach einmaliger Verwendung im Archiv verschwindet, wurde für 2013 eine Datenbank mit sämtlichen als Bild- oder Tondatei verfügbaren Prüfungsreden angelegt. Die Datenbank ist nach Leistungsniveau, 
Ausgangsprachen und Prüfungszeitpunkt durchsuchbar und enthält mittlerweile über 200 Reden in zwölf Sprachen. Die jeweils jüngsten Reden werden im Folgejahr für Probeläufe als Prüfungsvorbereitung verwendet, womit jede aufgezeichnete Rede gleich drei Mal genutzt wird: Zuerst als Prüfungsrede, ein Jahr später als Mock-Exam und in den Folgejahren als Übungsmaterial. Dieser Fundus an hochwertigen Reden wird von den Studierenden rege genutzt.

\section{Flexibilität im Angebot}

Als eine von wenigen Hochschulen bietet die ZHAW die Anpassung der Sprachenpalette an die Bedürfnisse der Studierenden an. So konnten etwa in den letzten Jahren auch seltenere Ausgangssprachen wie Tschechisch, Finnisch oder Chinesisch sowie neu die Muttersprachen Englisch und Russisch angeboten werden. Die Departementsleitung und die Vertiefungsleitung sehen diese Flexibilität als Wettbewerbsvorteil, da eine seltene Ausgangsprache in der Regel die Attraktivität unserer AbsolventInnen für zukünftige Arbeitgeber erhöht und uns als Ausbildungsstätte attraktiv macht. Insbesondere auf Seiten der EU und anderer internationaler Institutionen ist man an einem solchen Angebot besonders interessiert.

Bei den Diplomprüfungen in Konferenzdolmetschen galt bis vor kurzem noch ein Prüfungsformat, das sich über die Jahrzehnte bewährt hatte, aber nur beschränkt eine marktnahe Dolmetschsituation simulierte. Aus diesem Grund beschloss die Vertiefungsleitung vor gut drei Jahren, das Prüfungsformat grundlegend zu überarbeiten und an die heutige Arbeitswelt anzupassen. Die Studierenden erhalten heute eine generelle Themenangabe sieben Tage vor der Prüfung und können sich mit den verfügbaren Hilfsmitteln professionell vorbereiten. Ausser bei der Simultanprüfung mit Textvorlage werden die Prüfungsreden nicht mehr vorgelesen, sondern frei vorgetragen. Zudem werden alle Prüfungsreden für die Abschlussprüfungen auf Video vorgängig aufgezeichnet. Dies ermöglicht nicht nur eine optimale Qualität des Prüfungsmaterials (inhaltlich und technisch), sondern auch eine bessere Vergleichbarkeit der Prüfungsleistungen. Die Rückmeldungen unserer externen PrüfungsexpertInnen sowie der BeobachterInnen der Europäischen Kommission und des Europäischen Parlaments bestätigen, dass dadurch die angestrebte Praxisnähe soweit bei Prüfungen möglich erreicht wurde.

Damit unsere Studierenden nicht nur in den Dolmetschlabors üben, sondern auch echte Konferenzsituationen erleben können, erhalten sie wiederholt Gelegenheit, im Rahmen von freiwilligen Einsätzen (Volontariaten) bei echten 
KundInnen $\mathrm{zu}$ dolmetschen, vorzugsweise bei Institutionen mit karitativem Zweck, namentlich Hilfswerken. Unsere Studierenden erwerben so eine Praxiserfahrung, die ihnen für die Anfänge ihrer Laufbahn Selbstvertrauen verleiht. Seit jüngster Zeit werden die Studierenden darüber hinaus in die Organisation dieser Dolmetschleistungen für VolontariatskundInnen aktiv eingebunden, wodurch sie lernen, die Anforderungen für ihre Tätigkeit selber zu kommunizieren und für ihren Auftrag Verantwortung zu übernehmen.

Neben diesen praktischen Dolmetscherfahrungen werden ab dem 2. Semester im Master Angewandte Linguistik wie schon früher Besuche bei potenziellen Arbeitgebern organisiert (z.B. CH-Parlament in Bern, EU-Parlament und -Kommission in Brüssel, Europäisches Patentamt in München). Zudem erhalten die Studierenden Gelegenheit, im Unterricht vorbesprochene Inhalte (z. B. Aktionärsversammlungen) direkt mitzuerleben: Die Studierenden dürfen ihre Lehrkräfte teilweise zu Aufträgen begleiten und ihnen beim Arbeiten über die Schulter schauen. Gelegentlich bietet sich auch die Möglichkeit, in einer sogenannten „stummen Kabine“ gleich mitzuüben.

Die im Rahmen eines Berichts der Studierenden geschilderten Erfahrungen und Lernprozesse am Ende des Studiums zeigen, dass diese Praxiserfahrungen bei den angehenden DolmetscherInnen nicht nur einen Denkprozess, sondern auch einen Entwicklungssprung auslösen, der mit dem Unterricht allein nicht zu erreichen wäre.

\section{Organisatorische Optimierung}

Um die verschiedenen Massnahmen zur Curriculumsgestaltung sowie zur Qualitätssteigerung vorzubesprechen und zu koordinieren, wurde 2012 aus den an der ZHAW fest angestellten DolmetscherInnen, externen KollegInnen, der Professorin für Dolmetschwissenschaft sowie der Vertiefungsleitung und -assistenz ein Training Committee gebildet. Dieser Ausschuss befindet über die Weiterbildung der Dozierenden, neue Prüfungsformate und -bestimmungen, Volontariate und technische Aspekte der Vertiefung. Er dient der Vertiefungsleitung somit als wichtiger Resonanzraum und Think-Tank. Er hat u. a. zur Zusammenstellung der im Studium zu erwerbenden Kompetenzen in Form eines Lehrplans geführt, der den Dozierenden als Referenzwerk dient, um die Entwicklung der Studierenden gegenüber den Lernzielen genauer mitverfolgen zu können. Auch den Studierenden hilft er, sich einen Gesamtüberblick über die verschiedenen Anforderungen und Lernziele zu verschaffen, die sonst nur 
einzeln für jeden Kurs standardmässig in Kursbeschreibungen festgehalten werden.

Interne Weiterbildungsveranstaltungen für Dolmetschdozierende bilden eine wesentliche Kompetenzerweiterung ihrer hauptsächlich praktischen Dolmetscherfahrung. Seit 2012 erarbeiten die Dolmetschdozierenden im Rahmen von ein bis zwei Mal im Jahr stattfindenden Workshops teilweise selber bestimmte Arbeitsgrundlagen, oder aber es werden ausgewiesene ExpertInnen aus der Praxis eingeladen, die zu unterrichtsrelevanten Themen sprechen. Die bisherigen Workshops widmeten sich unter anderem der Entwicklung des Lehrplans, der Dolmetschwissenschaft und Weitergabe wissenschaftlicher Erkenntnisse, der zielführenden Gestaltung von Unterrichtsstunden sowie der Strukturierung und Präsentation von Feedback. Die Workshops stehen immer auch Dozierenden aus den anderen Vertiefungen sowie aus dem BA-Programm offen. Dies ermöglicht eine Verankerung der überwiegend externen Dolmetschdozierenden am Departement Angewandte Linguistik.

Eine besondere Eigenart des Masters in Angewandter Linguistik ist das sogenannte Rahmenstudium, dessen Kurse für die Studierenden aller drei Vertiefungen (Konferenzdolmetschen, Fachübersetzen, Organisationskommunikation) gemeinsam angeboten werden. In dieser Struktur werden nicht nur gemeinsame Grundlagen der Angewandten Linguistik und wissenschaftlichen Methodik erlernt, es erfolgt auch ein Austausch zwischen den später im Arbeitsleben zusammenarbeitenden Berufsgruppen. Um diese Zusammenarbeit so konkret wie möglich zu gestalten, findet seit $2015 \mathrm{im}$ 2. Semester MA AL neben dem theoretischen Unterricht in Translationswissenschaft und Organisationskommunikation ein gemeinsames Projekt aller drei Vertiefungen statt, in dem jede Gruppe ihre Kernkompetenzen wahrnimmt sowie ihr Wissen und Können zum Erfolg des Projekts beisteuert. Die Studierenden konzipieren, planen und verwirklichen gemeinsam eine öffentlich zugängliche grössere Veranstaltung zu einem von der Studiengangleitung abgesteckten Themenbereich.

Die ersten Erfahrungen mit diesem Studentenprojekt sind sehr ermutigend. Die im Dezember 2015 und 2016 durchgeführten Podiumsdiskussionen waren äusserst gehaltvoll, ausgezeichnet organisiert sowie umfassend dokumentiert und damit für alle Beteiligten eine Bereicherung. Aufbauend auf einem theoretischen Fundament, praktischer Erfahrung und gegenseitigem Austausch leistet der Master in Angewandter Linguistik somit einen Beitrag zu einer reibungslosen Zusammenarbeit der verschiedenen Akteure im späteren Berufsleben. 


\section{Ein Blick in die Zukunft}

KonferenzdolmetscherInnen werden heute an der ZHAW professioneller denn je ausgebildet. Die positiven Rückmeldungen namhafter Akteure auf dem Markt belegen, dass die Anpassung und Modernisierung der Ausbildung über die letzten Jahre wesentlich dazu beigetragen hat, dass viele unserer AbsolventInnen schon bald nach dem Studium mit regelmässigen Dolmetschaufträgen rechnen können und dank ihrer Ausbildung ein Auskommen finden.

Die Zukunft für KonferenzdolmetscherInnen wird indes nicht einfach: Sparzwänge die zunehmende Verbreitung des Gebrauchs von Englisch als Lingua franca sowie das Vordringen von KollegInnen aus Märkten mit niedrigeren Honoraren (namentlich Deutschland) in die Schweiz verschärfen die Konkurrenzsituation. Parallel dazu versuchen branchenfremde Technologie-Anbieter, Konferenzveranstalter dazu zu bringen, auf Remote-Lösungen zu setzen und auf Dolmetschkabinen und DolmetscherInnen vor Ort zu verzichten. Sollten sich solche Lösungen etablieren, würde eine persönliche, vor Ort erbrachte und hochwertige Expertendienstleistung $\mathrm{zu}$ einer anonymen, herkunftslosen und austauschbaren Massenware degradiert.

Die ZHAW tritt diesen Entwicklungen mit einem bewährten Konzept entgegen, das an den Grundsätzen des selbstgesteuerten, an der professionellen Praxis ausgerichteten und zugleich forschungsgeleiteten und wissenschaftsbasierten Lernens ausgerichtet ist. Über die ständige Weiterentwicklung des Lehrangebots ermöglicht sie ihren Studierenden eine gründliche Vorbereitung auf die anstehenden Herausforderungen und vermittelt den angehenden DolmetscherInnen das Rüstzeug, um sich als Premium-Anbieter mit klarem Bekenntnis zu Qualität und berufsethischen Prinzipien zu positionieren.

\section{Literaturverzeichnis}

Albl-Mikasa, M. (2017). ELF and Translation/Interpreting. In Jenkins, J., Baker, W. \& Dewey, M. (Hrsg.). The Routledge Handbook of English as a Lingua Franca. London/New York: Routledge.

Albl-Mikasa, M., Bartels, L., Mohler, L., \& Wick, B. (2017). World Englishes and English as a lingua franca in interpreter training. In Hagemann, S., Kempa, T., Neu, J. \& Walter, S. (Hrsg.). Translationslehre und Bologna-Prozess: Unterwegs zwischen Einheit und Vielfalt / Translation/Interpreting Teaching and the Bologna Process: Pathways between Unity and Diversity. TransüD 87 (S. 217-249). Berlin: Frank und Timme.

Kalina, S. (2017). Bologna - viele Chancen und ein paar Fallstricke für Dolmetschstudiengänge. In Hagemann, S., Kempa, T., Neu, J. \& Walter, S. (Hrsg.). Translationslehre und BolognaProzess: Unterwegs zwischen Einheit und Vielfalt / Translation/Interpreting Teaching and the Bologna Process: Pathways between Unity and Diversity. TransÜD 87 (S. 51-76). Berlin: Frank und Timme. 